\title{
Thermo-mechanical vibration of short carbon nanotubes embedded in pasternak foundation based on nonlocal elasticity theory
}

\author{
B. Amirian ${ }^{\mathrm{a}}$, R. Hosseini-Ara ${ }^{\mathrm{b}, *}$ and H. Moosavi ${ }^{\mathrm{a}}$ \\ ${ }^{a}$ Department of Mechanical Engineering, Isfahan University of Technology, Isfahan, Iran \\ ${ }^{\mathrm{b}}$ Department of Engineering, Payame Noor University, Tehran, Iran
}

Received 8 June 2012

Revised 13 November 2012

Accepted 2 March 2013

\begin{abstract}
This study is concerned with the thermal vibration analysis of a short single-walled carbon nanotube embedded in an elastic medium based on nonlocal Timoshenko beam model. A Winkler- and Pasternak-type elastic foundation is employed to model the interaction of short carbon nanotubes and the surrounding elastic medium. Influence of all parameters such as nonlocal small-scale effects, high temperature change, Winkler modulus parameter, Pasternak shear parameter, vibration mode and aspect ratio of short carbon nanotubes on the vibration frequency are analyzed and discussed. The present study shows that for high temperature changes, the effect of Winkler constant in different nonlocal parameters on nonlocal frequency is negligible. Furthermore, for all temperatures, the nonlocal frequencies are always smaller than the local frequencies in short carbon nanotubes. In addition, for high Pasternak modulus, by increasing the aspect ratio, the nonlocal frequency decreases. It is concluded that short carbon nanotubes have the higher frequencies as compared with long carbon nanotubes.
\end{abstract}

Keywords: Short carbon nanotubes, vibration, pasternak effect, high temperature change, nonlocal timoshenko beam

\section{Introduction}

After seminal work of Iijima [1], many researchers have great interests on dynamic analysis of carbon nanotubes (CNTs), because of their exceptional mechanical, electrical and thermal properties [2-4]. These properties of CNTs lead to its application in the fields of nano-electronics, nano-devices, nano-composites, etc. It has been shown that the CNTs with extremely high elastic modulus and low mass density can serve as terahertz nano-resonators [5-8] in nano-electro-mechanical systems (NEMS). For example, they are thermally stable up to $2800^{\circ} \mathrm{C}$ in vacuum, with a thermal conductivity which is twice as large as diamond, and having an electric- current- carrying about 1000 times greater than copper wire [9].

On the other hand, most applications tend to shrink the dimensions of nano-scale devices. Researchers show that short nanotubes with open ends are required to overpass the diffusion limitation [10]. Wang et al. [11] studied the electro-chemical behavior of ultra-short carbon nanotubes. They concluded that compared with conventional long CNTs, short CNTs show much better electro-chemical performances. Lopez et al. [12] discussed structural and thermal stability of short carbon nanotubes. Seidel et al. [13] showed that short CNTs, with the lengths less than

\footnotetext{
${ }^{*}$ Corresponding author: R. Hosseini-Ara, Department of Engineering, Payame Noor University, PO BOX 19395-3697 Tehran, Iran. E-mail: hosseiniara@gmail.com.
} 
20 nanometers, are useful in molecular electronics and CNT field-effect transistors (CNTFETs). Yoon et al. [14] studied the vibration behavior of multi-walled carbon nanotubes embedded in an elastic medium using multipleelastic beam model. Zhang et al. [15] developed a double-elastic beam model for transverse vibration of doublewalled carbon nanotubes (DWCNTs) under axial compressive load using Euler-Bernoulli beam theory. A nonlocal elastic model for static and dynamic analysis of carbon nanotubes embedded in two-parameter Pasternak foundation applied by Ref. [16-19]. In fact, the Winkler-type elastic foundation is approximated as a series of closely spaced, mutually independent, vertical linear elastic springs. The interaction between the springs is ignored in Winkler-type foundations. Therefore, a more realistic and generalized representation of the elastic medium can be accomplished by a two-parameter foundation model [20]. However, only a limited portion of literature is concerned with the vibration analysis of carbon nanotubes considering the thermal effect and Pasternak foundation.

Moreover, the size-dependent nonlocal continuum theory is used because at small-scale length, the material microstructures, such as lattice spacing between individual atoms, become increasingly significant and thus its effect can no longer be ignored [21]. In addition, most of the previous studies treated a nanotube as a simple Euler beam and neglected the effects of transverse shear deformation and rotary inertia. In fact, the Euler-Bernoulli beam models holds only when the length of the beam is much larger than its thickness (i.e., more than 10 times). However, in many applications the length of the nanotube is insufficiently long to be simplified as an Euler beam and the influence of shear deformation and rotary inertia should be taken into account [22].

In this paper, we present a nonlocal Timoshenko beam model and derive all governing equations and boundary conditions for vibration analysis of short carbon nanotubes, using Hamilton's principle. The surrounding elastic medium is described as the Winkler- and Pasternak type foundation. Therefore, an analytically solution is used to obtain the natural frequency of short carbon nanotubes with immoveable supports. Finally, the influences of nonlocal parameter, Winkler and Pasternak shear modulus parameter, high temperature change, aspect ratio and vibration mode on vibration of short carbon nanotubes are discussed.

\section{Nonlocal nanobeam model for linear analysis of short CNTs}

The nonlocal elasticity theory is developed by Eringen [23,24] and Eringen and Edelen [25]. According to theory of nonlocal elasticity, the stress at a point $x$ in a body depends not only on the strain at point $x$ (hyper elastic case) but also on those at all other points of the body. Thus the nonlocal stress tensor $\sigma$ at point $x$ is expressed as

$$
\begin{aligned}
& \sigma_{i j}=\int_{V} \lambda\left(\left|x^{\prime}-x\right|, \tau\right) \varepsilon_{k l}\left(x^{\prime}\right) C_{i j k l} d V\left(x^{\prime}\right), \\
& \left(1-\tau^{2} l^{2} \nabla^{2}\right) \sigma=t, \tau=\frac{e_{0} a}{l} .
\end{aligned}
$$

The terms $\sigma_{i j}, \varepsilon_{k l}$ and $C_{i j k l}$ are the stress, strain and fourth order elasticity tensor, respectively. $\lambda\left(\left|x^{\prime}-x\right|, \tau\right)$ is the Kernel function or nonlocal modulus or attenuation function incorporating into constitutive equations, $\left|x^{\prime}-x\right|$ represents the distance in Euclidean form, and $\tau$ is the material constant that depends on the internal (e.g. lattice parameter, granular size, distance between $\mathrm{C}-\mathrm{C}$ bonds) and external characteristic length (e.g. wave length). The values of $a$ and $l$ are the internal and external lengths, respectively, and classical stress tensor is defined as $t=C: \varepsilon$, where ' $:$ ' represents the double dot product. The parameter $e_{0}$ is estimated such that the relations of the nonlocal elasticity model could provide satisfactory approximation of atomic dispersion curves of plane waves with those of atomic lattice dynamics [26]. In this regard, Zhang et al. [27] performed analysis of elastic interactions between Stone-Wales and divacancy defects on carbon graphene sheets. They concluded that the displacement field around defects obtained from the nonlocal continuum models and MD can match very well if $e_{0}$ is chosen to be 8.79. Duan et al. [28] reported the value of $e_{0}$ ranging from 0-19 for carbon nanotubes with nonlocal Timoshenko beam theory and using MD results. Wang studied the wave propagation in carbon nanotubes with two nonlocal continuum mechanics models: elastic Euler-Bernoulli and Timoshenko beam models and proposed $e_{0}=1 \sim 14$ [29]. Shen and Zhang [30] considered $e_{0}$ in the range of 3 to 5.1 and estimated small scale effect parameter by matching the buckling torque of CNTs observed from the MD simulation results with the numerical results obtained from the nonlocal shear deformable shell model. Chan and Zhao [31] reported $e_{0}=0.23$ by considering nonlocal elasticity 
as an important factor in the spinning CNTs. On the other hand, Khademolhosseini et al. [32] proposed $e_{0}=$ 0.18 through comparison of the MD simulation results with classical and nonlocal dispersion relations. Wang et al. [33] proposed that $e_{0}=0.288$ be used in determination of the dispersion curves via elastic beam theories and the Molecular Dynamics method. In addition, Eringen [23] proposed $e_{0}$ as 0.39 . On the other hand, Eringen also proposed $e_{0}=0.31$ based on the comparison of Rayleigh surface wave via nonlocal continuum mechanics and lattice dynamics. Zhang et al. [34] approximated that $e_{0}=0.82$ by matching the theoretical buckling strain obtained by the nonlocal elastic cylindrical shell model. It is clear that a large range of values for scale parameter, $e_{0} a$, is possible. The above mentioned studies indicate that reasonable choice of the value of the parameter $e_{0} a$ is crucial to ensure the validity of the nonlocal models. Although, $e_{0}$ is a key parameter in the nonlocal elasticity theory, there is hitherto no rigorous study being made on estimating the scaling parameter for various physical problems. Therefore, more works, especially experimental tests, are required to determine $e_{0}$ more accurately for CNTs. In this study, the small scale coefficients were taken as $e_{0} a=0.0,0.5,1.0,1.5,2.0(\mathrm{~nm})$ for carbon nanotubes as described by Ref. [35].

\section{Formulations}

The displacement field equation based on Timoshenko beam theory is given as

$$
\begin{aligned}
& u_{1}(x, y, z, t)=u(x, t)+z \varphi(x, t) \\
& u_{2}(x, y, z, t)=0 \\
& u_{3}(x, y, z, t)=w(x, t)
\end{aligned}
$$

where $u_{1}$ and $u_{3}$ are the axial and transverse displacement of the point $(x, 0)$ on the mid-plane (i.e., $\left.z=0\right)$ of the beam and $\varphi(x, t)$ denotes the rotation of the cross-section beam. The nonzero strains according to Timoshenko beam theory are expressed as

$$
\varepsilon_{x x}=\frac{\partial u(x, t)}{\partial x}+z \frac{\partial \varphi(x, t)}{\partial x}, \varepsilon_{x z}=\frac{\partial w(x, t)}{\partial x}+\varphi(x, t),
$$

where $\varepsilon_{x x}$ and $\varepsilon_{x z}$ are the axial and shear strain, respectively. The equations of motion of the nonlocal SWCNTs embedded in an elastic medium can be derived from the Hamilton's principle

$$
\delta \int_{0}^{t_{1}}[K-(U+V)] d t=0
$$

The strain energy of beam, $U$ is given by

$$
U=\frac{1}{2} \int_{0}^{L}\left\{N \frac{\partial u(x, t)}{\partial x}+M \frac{\partial \varphi(x, t)}{\partial x}+Q\left(\frac{\partial w(x, t)}{\partial x}+\varphi(x, t)\right)\right\} d x
$$

where the normal resultant force $N$, bending moment $M$ and transverse shear force $Q$ are calculated from

$$
N=\int_{A} \sigma_{x x} d A, \quad M=\int_{A} \sigma_{x x} z d A, \quad Q=\int_{A} \sigma_{x z} d A
$$

The general form of kinetic energy comes in the form below

$$
K=\int_{0}^{L}\left\{\frac{\rho A}{2}\left(\frac{\partial u(x, t)}{\partial t}\right)^{2}+\frac{\rho I}{2}\left(\frac{\partial \varphi(x, t)}{\partial t}\right)^{2}+\frac{\rho A}{2}\left(\frac{\partial w(x, t)}{\partial t}\right)^{2}\right\} d x
$$


where $\rho$ is the mass density of beam material, $A$ is the beam's cross sectional area (circular-cross section) and $I$ is the second moment of area about $y$-axis. The potential energy is equal to work done by external forces and is given by

$$
V=W_{E}=-\frac{1}{2} \int_{0}^{L}\left\{f(x, t) u(x, t)+q(x, t) w(x, t)+\bar{N}\left(\frac{\partial w(x, t)}{\partial x}\right)^{2}+f_{e} w(x, t)\right\} d x
$$

Indeed, the Eq. (9) is extended form of potential energy in Ref. [36]. In this equation, the effects of thermal field and two-parameter elastic medium are considered. In above equation, negative sign indicates that work is done on the body, $f(x, t)$ and $q(x, t)$ are the axial and transverse distributed forces (measured per unit length), $\bar{N}$ is applied compressive force and $f_{e}$ is the density of reaction force of elastic foundation and expressed as

$$
f_{e}=K_{W} w(x, t)-K_{G} \frac{\partial^{2} w(x, t)}{\partial x^{2}}
$$

The terms $K_{W}$ and $K_{G}$ represent the Winkler and shear modulus (shear layer foundation stiffness) of the elastic medium, respectively. By using calculus of variation and substituting Eqs (6), (8) and (9) into Eq. (5), the Hamilton's principle can be represented as

$$
\begin{aligned}
0= & \int_{0}^{t} \int_{0}^{L}\left\{m_{0}\left(\frac{\partial u}{\partial t} \frac{\partial \delta u}{\partial t}+\frac{\partial w}{\partial t} \frac{\partial \delta w}{\partial t}\right)+m_{2} \frac{\partial \varphi}{\partial t} \frac{\partial \delta \varphi}{\partial t}-N \frac{\partial \delta \varphi}{\partial x}-M \frac{\partial \delta w}{\partial x}\right. \\
& \left.-Q\left(\frac{\partial \delta w}{\partial x}+\partial \delta \varphi\right)+f(x, t) \delta u+q(x, t) \delta w+\bar{N}\left(\frac{\partial w}{\partial x} \frac{\partial \delta w}{\partial x}\right)+f_{e} \delta w\right\} d x d t
\end{aligned}
$$

The mass inertia $m_{0}$ and $m_{2}$ are defined by

$$
m_{0}=\int_{A} \rho d A=\rho A, \quad m_{2}=\int_{A} \rho z^{2} d A=\rho I
$$

With integrating by parts of Eq. (11) setting the coefficient of $\delta u, \delta w$ and $\delta \varphi$ to zero leads to equations of motion

$$
\begin{aligned}
& \frac{\partial N}{\partial x}+f(x, t)=m_{0} \frac{\partial^{2} u}{\partial t^{2}} \\
& \frac{\partial Q}{\partial x}+q(x, t)-K_{W} w+K_{G} \frac{\partial^{2} w}{\partial x^{2}}-\bar{N} \frac{\partial^{2} w}{\partial x^{2}}=m_{0} \frac{\partial^{2} w}{\partial t^{2}} \\
& \frac{\partial M}{\partial x}-Q=m_{2} \frac{\partial^{2} \varphi}{\partial t^{2}} .
\end{aligned}
$$

It is assumed that the axial and transverse distributed forces are equal to zero

$$
f(x, t)=q(x, t)=0
$$

Differentiating Eq. (13c) once related to $x$ and substituting into Eq. (13b), we obtain the nonlocal bending moment, $M$, and shear force, $Q$, in the Timoshenko beam theory

$$
\begin{aligned}
& M=E I \frac{\partial \varphi}{\partial x}+\mu\left[\bar{N} \frac{\partial^{2} w}{\partial x^{2}}+K_{W} w-K_{G} \frac{\partial^{2} w}{\partial x^{2}}+m_{0} \frac{\partial^{2} w}{\partial t^{2}}+m_{2} \frac{\partial^{3} \varphi}{\partial x \partial t^{2}}\right] \\
& Q=K_{S} G A\left(\frac{\partial w}{\partial x}+\varphi\right)+\mu \frac{\partial}{\partial x}\left[\bar{N} \frac{\partial^{2} w}{\partial x^{2}}+K_{W} w-K_{G} \frac{\partial^{2} w}{\partial x^{2}}+m_{0} \frac{\partial^{2} w}{\partial t^{2}}\right]
\end{aligned}
$$


where $E$ is the Young's modulus, $G$ is the shear modulus or modulus of rigidity and $K_{s}$ is the Timoshenko's shear correction that accounts for non-uniform shear stress distribution through the thickness of the beam. By substituting Eqs (15) and (16) into Eq. (11), we obtain the complete form of equations of motion

$$
\begin{aligned}
& E A \frac{\partial^{2} u}{\partial x^{2}}+\mu m_{0}\left(\frac{\partial^{4} u}{\partial x^{2} \partial t^{2}}\right)=m_{0} \frac{\partial^{2} u}{\partial t^{2}} \\
& E I \frac{\partial^{2} \varphi}{\partial x^{2}}-K_{S} G A\left(\frac{\partial w}{\partial x}+\varphi\right)+\mu m_{2} \frac{\partial^{4} \varphi}{\partial x^{2} \partial t^{2}}=m_{2} \frac{\partial^{2} \varphi}{\partial t^{2}} \\
& \frac{\partial}{\partial x}\left[K_{S} G A\left(\frac{\partial w}{\partial x}+\varphi\right)\right]+K_{W} w-K_{G} \frac{\partial^{2} w}{\partial x^{2}}-\bar{N} \frac{\partial^{2} w}{\partial x^{2}} \\
& \quad+\mu \frac{\partial^{2}}{\partial x^{2}}\left[\bar{N} \frac{\partial^{2} w}{\partial x^{2}}+K_{W} w-K_{G} \frac{\partial^{2} w}{\partial x^{2}}+m_{0} \frac{\partial^{2} w}{\partial t^{2}}\right]=m_{0} \frac{\partial^{2} w}{\partial t^{2}}
\end{aligned}
$$

The boundary conditions of Eqs $(17 \mathrm{a}-\mathrm{c})$ are written as follows:

$$
\begin{aligned}
& \left.\left(E A \frac{\partial u}{\partial x}+\mu m_{0} \frac{\partial^{3} u}{\partial x \partial t^{2}}\right) \delta u\right|_{0} ^{L}=0 \\
& \left.\left\{K_{S} G A\left(\frac{\partial w}{\partial x}+\varphi\right)-\bar{N} \frac{\partial w}{\partial x}+\mu \frac{\partial}{\partial x}\left[\bar{N} \frac{\partial^{2} w}{\partial x^{2}}+K_{W} w-K_{G} \frac{\partial^{2} w}{\partial x^{2}}+m_{0} \frac{\partial^{2} w}{\partial t^{2}}\right]\right\} \delta \varphi\right|_{0} ^{L}=0 \\
& \left.\left\{E I \frac{\partial \varphi}{\partial x}+\mu\left[\bar{N} \frac{\partial^{2} w}{\partial x^{2}}+K_{W} w-K_{G} \frac{\partial^{2} w}{\partial x^{2}}+m_{0} \frac{\partial^{2} w}{\partial t^{2}}+m_{2} \frac{\partial^{3} \varphi}{\partial x \partial t^{2}}\right]\right\} \delta w\right|_{0} ^{L}=0 .
\end{aligned}
$$

Here, $\bar{N}$ represents the axial force on the CNTs and is expressed as

$$
\bar{N}=N_{m}+N_{\theta}
$$

where $N_{m}$ is the axial force due to the mechanical loading prior to buckling and $N_{\theta}$ is the axial force due to the influence of temperature change. Here, the theory of thermal elasticity mechanics is adopted because the Young's modulus of SWCNT is insensitive to temperature change in the tube at temperatures of less than approximately $1100^{\circ} \mathrm{K}$, but decreases at high temperature [37]. In addition, the high thermal conductivity of CNTs leads to the uniform and constant axial force, $N_{\theta}$, as below [38]

$$
N_{\theta}=-\frac{E A}{1-2 v} \alpha_{x} \theta
$$

where $\alpha_{x}$ is the coefficient of thermal expansion in the direction of $x$-axis, $v$ is the Poisson's ratio and $\theta$ denotes the change in temperature. Here changes for high temperature environment will be considered. By considering the boundary conditions for immoveable supports, $u(0, t)=u(L, t)=0$, the axial force due to mechanical loading will be zero.

\section{Analytical solution for vibration of short carbon nanotubes}

By the application of the separation of variables, we can assume periodic solutions of the form $\varphi(x, t)=\phi(x) e^{i \omega t}$ and $w(x, t)=W(x) e^{i \omega t}$ for vibration analysis of short carbon nanotubes, where $\phi(x)$ and $W(x)$ are the mode shapes and $\omega$ is the frequency of natural vibration [36]. Therefore, with substituting $\varphi(x, t)$ and $w(x, t)$ into Eqs (17b) and (17c), we obtain

$$
\frac{d}{d x}\left(E I \frac{d \phi}{d x}\right)-K_{S} G A\left(\frac{d W}{d x}+\phi\right)+m_{2} \omega^{2}\left(\phi-\mu \frac{d^{2} \phi}{d x^{2}}\right)=0
$$




$$
\begin{aligned}
& \frac{d}{d x}\left[K_{S} G A\left(\frac{d W}{d x}+\phi\right)\right]+K_{W} W-K_{G} \frac{d^{2} W}{d x^{2}}-\bar{N} \frac{d^{2} W}{d x^{2}}+\mu \bar{N} \frac{d^{4} W}{d x^{4}}+\mu K_{W} \frac{d^{2} W}{d x^{2}} \\
& \quad-\mu K_{G} \frac{d^{4} W}{d x^{4}}-\mu m_{0} \omega^{2} \frac{d^{2} W}{d x^{2}}+m_{0} \omega^{2} W=0 .
\end{aligned}
$$

Differentiating Eq. (24) once, substituting for $\frac{d \phi}{d x}$ and by some simplifications, we obtain

$$
A \frac{d^{6} W}{d x^{6}}+B \frac{d^{4} W}{d x^{4}}+C \frac{d^{2} W}{d x^{2}}+D W=0
$$

where

$$
\begin{aligned}
A= & \mu\left(E I-\mu m_{2} \omega^{2}\right)\left(\frac{\bar{N}-K_{G}}{K_{S} G A}\right) \\
B= & \left(E I-\mu m_{2} \omega^{2}\right)\left(1-\frac{\bar{N}+K_{G}-\mu K_{W}+\mu m_{0} \omega^{2}}{K_{S} G A}\right) \\
& +\mu\left(\frac{\bar{N}-K_{G}}{K_{S} G A}\right)\left(m_{2} \omega^{2}-K_{S} G A\right) \\
C= & \left(m_{2} \omega^{2}-K_{S} G A\right)\left(1-\frac{\bar{N}+K_{G}-\mu K_{W}+\mu m_{0} \omega^{2}}{K_{S} G A}\right)+K_{S} G A \\
& +\left(E I-\mu m_{2} \omega^{2}\right)\left(\frac{m_{0} \omega^{2}+K_{W}}{K_{S} G A}\right) \\
D= & \left(m_{2} \omega^{2}-K_{S} G A\right)\left(\frac{m_{0} \omega^{2}+K_{W}}{K_{S} G A}\right) .
\end{aligned}
$$

It is noticeable that by ignoring the thermal and elastic medium parameters, the governing differential equation in Ref. [36] is derived. Considering the boundary conditions for simply-supported short-SWCNT with immoveable ends as

$$
w(0, t)=w(L, t)=0, \quad M=0 \text { at } x=0 \text { and } x=L .
$$

By substituting $\varphi(x, t)$ and $w(x, t)$ into Eqs (19) and (20), the natural boundary conditions for linear vibration are derived as below

$$
\begin{aligned}
& \left.\left\{K_{S} G A\left(\frac{d W}{d x}+\phi\right)-\bar{N} \frac{d W}{d x}+\mu \bar{N} \frac{d^{3} W}{d x^{3}}-\mu m_{0} \omega^{2} \frac{d W}{d x}\right\}\right|_{0} ^{L}=0 . \\
& \left.\left\{E I \frac{d \phi}{d x}+\mu\left[\bar{N} \frac{d^{2} W}{d x^{2}}+K_{W} W-K_{G} \frac{d^{2} W}{d x^{2}}-m_{0} \omega^{2} W-m_{2} \omega^{2} \frac{d \phi}{d x}\right]\right\}\right|_{0} ^{L}=0 .
\end{aligned}
$$

The general solution can be considered as follows:

$$
W(x)=\sum_{m=1}^{\infty} \sin \frac{m \pi}{L} x,
$$

where $m$ is the mode number. The above solution can satisfy all boundary conditions. With substituting Eqs (26) and (29) into Eq. (25), we calculate the natural frequencies for different cases. The frequency equation in general 


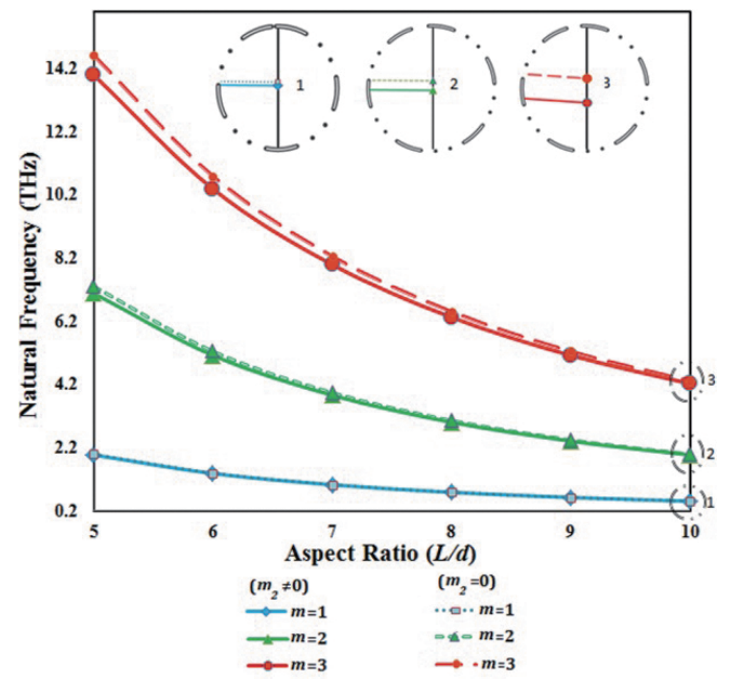

Fig. 1. Variation of natural frequency with aspect ratio $L / d$ for different change in mode numbers, $m$, with/without rotary inertia, $m_{2}$, for short-SWCNT, $\left(K_{W}=K_{G}=\bar{N}=0\right)$.

form is written as follows:

$$
\begin{aligned}
& -\left[\mu\left(E I-\mu m_{2} \omega^{2}\right)\left(\frac{\bar{N}-K_{G}}{K_{S} G A}\right)\right]\left(\frac{m \pi}{L}\right)^{6} \\
& +\left[\left(E I-\mu m_{2} \omega^{2}\right)\left(1-\frac{\bar{N}+K_{G}-\mu K_{W}+\mu m_{0} \omega^{2}}{K_{S} G A}\right)\right. \\
& \left.+\mu\left(\frac{\bar{N}-K_{G}}{K_{S} G A}\right)\left(m_{2} \omega^{2}-K_{S} G A\right)\right]\left(\frac{m \pi}{L}\right)^{4}-\left[\left(E I-\mu m_{2} \omega^{2}\right)\left(\frac{m_{0} \omega^{2}+K_{W}}{K_{S} G A}\right)\right. \\
& \left.+K_{S} G A+\left(m_{2} \omega^{2}-K_{S} G A\right) \times\left(1-\frac{\bar{N}+K_{G}-\mu K_{W}+\mu m_{0} \omega^{2}}{K_{S} G A}\right)\right]\left(\frac{m \pi}{L}\right)^{2} \\
& +\left(m_{2} \omega^{2}-K_{S} G A\right)\left(\frac{m_{0} \omega^{2}+K_{W}}{K_{S} G A}\right)=0 .
\end{aligned}
$$

\section{Numerical results}

Here, we present numerical solutions for the vibration of short-SWCNTs, considering the effects of thermal field and Pasternak elastic medium. The following values of effective properties are used [36]

$$
\rho=2300 \mathrm{Kg} / \mathrm{m}^{3}, E=1000 \mathrm{Gpa}, d=1 \times 10^{-9} \mathrm{~m}, K_{S}=0.877, v=0.19, G=420 \mathrm{Gpa}
$$

Here, we consider the aspect ratios (length-to-diameter, $L / d$ ) of short carbon nanotubes in the range of 5 to 10 . In the following, five different cases are studied in order to consider the different parameters:

Case (1). $\boldsymbol{\mu}=\mathbf{0}, \boldsymbol{K}_{G}=\mathbf{0}, \boldsymbol{K}_{W}=\mathbf{0}, \bar{N}=\mathbf{0}$

In this case, the natural frequency for local problem is calculated. The effect of aspect ratio and rotary inertia in local frequency is shown in Fig. 1. It depicts that with increasing in aspect ratio $(L / d)$, the natural frequencies decrease but with increasing in mode number $(m)$, the natural frequencies increase. Moreover, the natural frequencies 


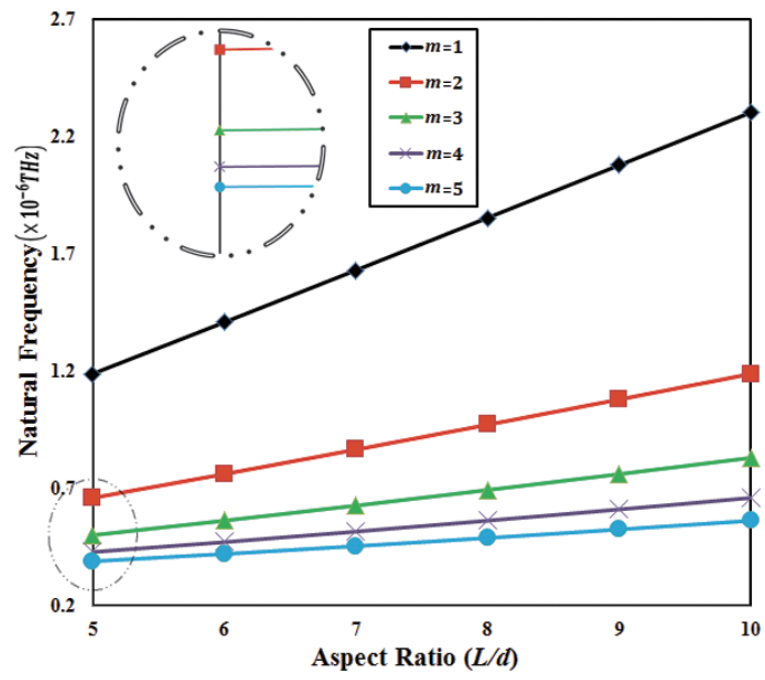

Fig. 3. Mode number effect on natural frequency of a short-SWCNT for different aspect ratio $L / d$ and $\mu=0.5$.

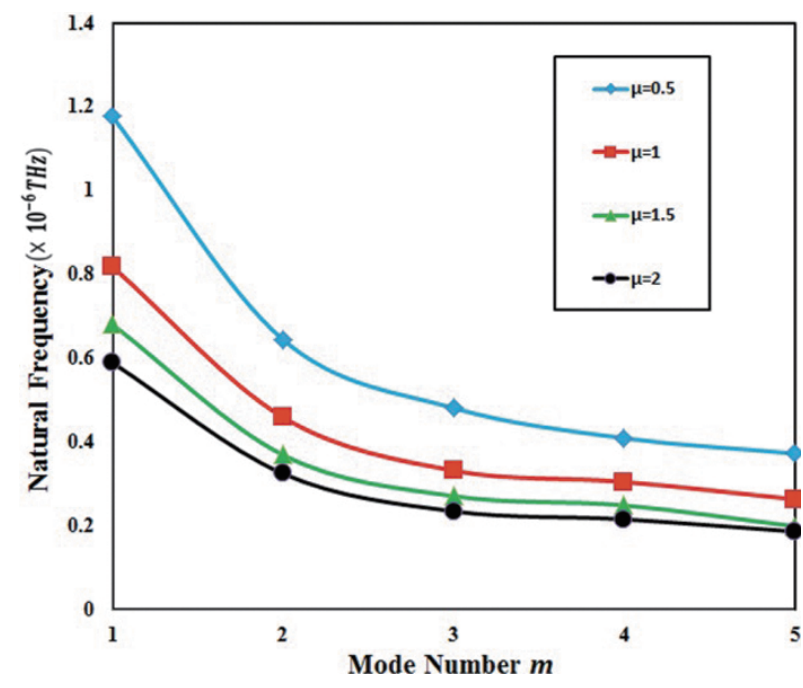

Fig. 5. The effect of mode number on natural frequency for different nonlocal parameter $\mu,\left(K_{W}=10^{3} \mathrm{~N} / \mathrm{m}^{2}, L / d=5\right)$.

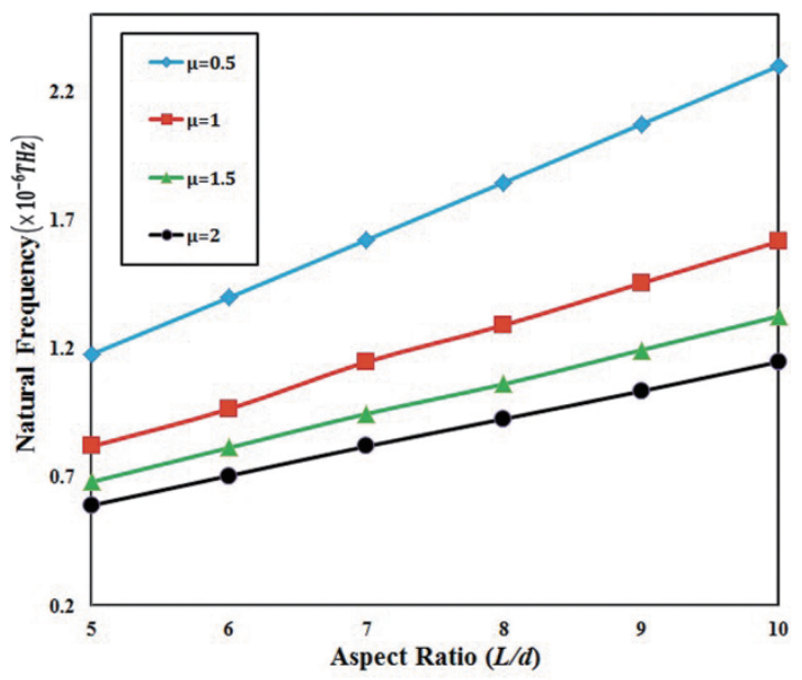

Fig. 4. Variation of natural frequency with aspect ratio $L / d$ for different nonlocal parameter, $\left(K_{W}=10^{3} \mathrm{~N} / \mathrm{m}^{2}, m=1\right)$.

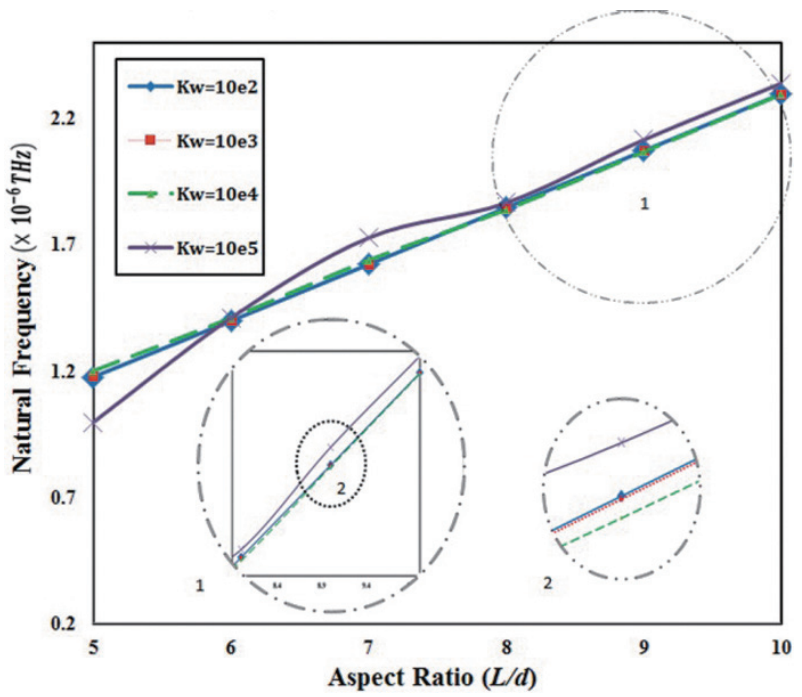

Fig. 6. The effect of Winkler modulus $K_{W}$ on natural frequency with different aspect ratio for $(m=1, \mu=0.5)$.

in the presence of rotary inertia are less than its counterpart without the effect of rotary inertia. Therefore, rotary inertia leads to diminish the amount of natural frequencies. This is the most basic case that has been considered.

Case (2). $\boldsymbol{K}_{\boldsymbol{G}}=\mathbf{0}, \boldsymbol{K}_{\boldsymbol{W}}=\mathbf{0}, \overline{\boldsymbol{N}}=\mathbf{0}$

In nonlocal cases in which the nonlocal parameter is considered, with increasing in aspect ratio, the amount of natural frequencies increase but it is lower than the local values (case1). The Winkler and shear modulus parameters are equal to zero. Also the effect of thermal field is not considered. Figure 2 shows the variation of natural frequencies for different nonlocal parameter. It is clear that increasing in nonlocal parameter $(\mu)$ in different aspect ratio leads to reducing natural frequency. In Fig. 3, the change of mode number with different aspect ratio has been considered. The nonlocal parameter is constant.

It can be shown that by increasing in mode number, unlike the previous case, the natural frequencies significantly reduce. 


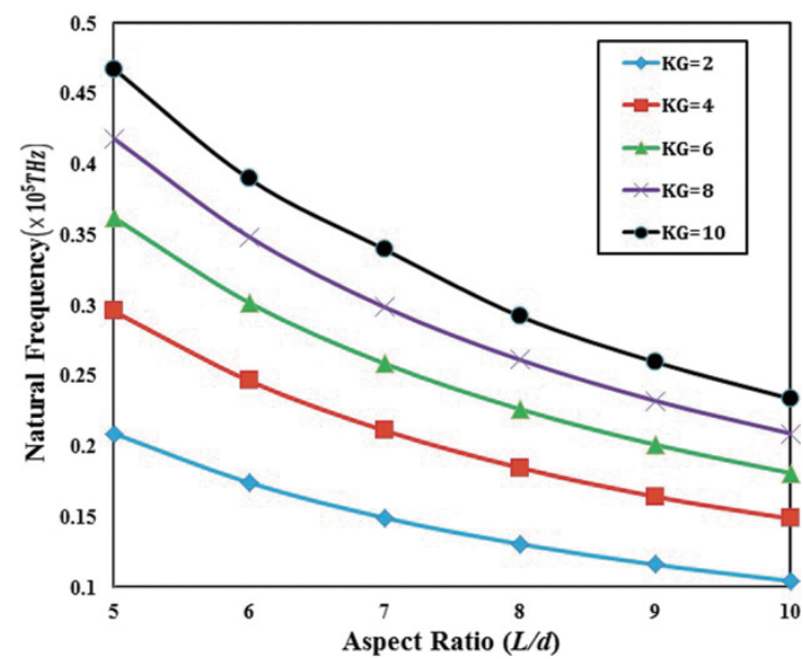

Fig. 7. The effect of shear modulus parameter $K_{G}$ on natural frequency of a short-SWCNT for different aspect ratio $L / d,\left(K_{W}=10^{2} \mathrm{~N} / \mathrm{m}^{2}\right.$, $m=1, \mu=0.5)$.

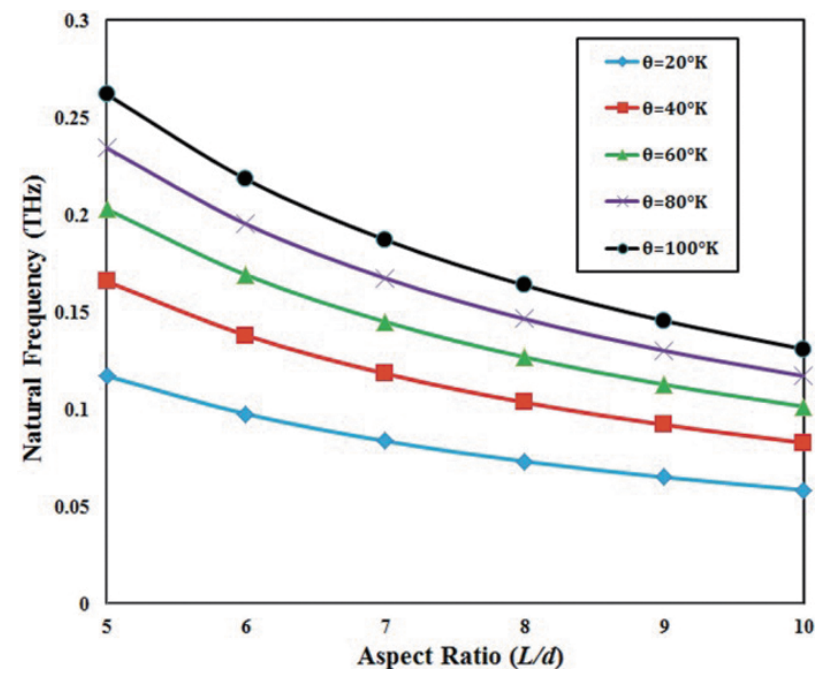

Fig. 9. The effect of temperature on frequency of a short-SWCNT with different aspect ratio $L / d,\left(K_{G}=K_{W}=0, m=1, \mu=0.5\right)$.

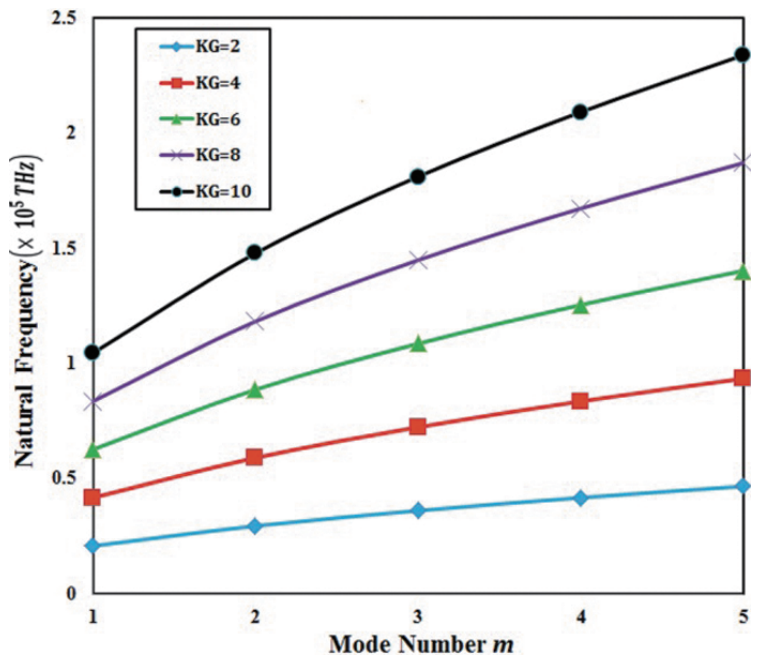

Fig. 8. The natural frequency of a short-SWCNT with different mode number $m$ and shear modulus parameter $K_{G},\left(K_{W}=10^{2} \mathrm{~N} / \mathrm{m}^{2}\right.$, $\mu=0.5, L / d=5)$.

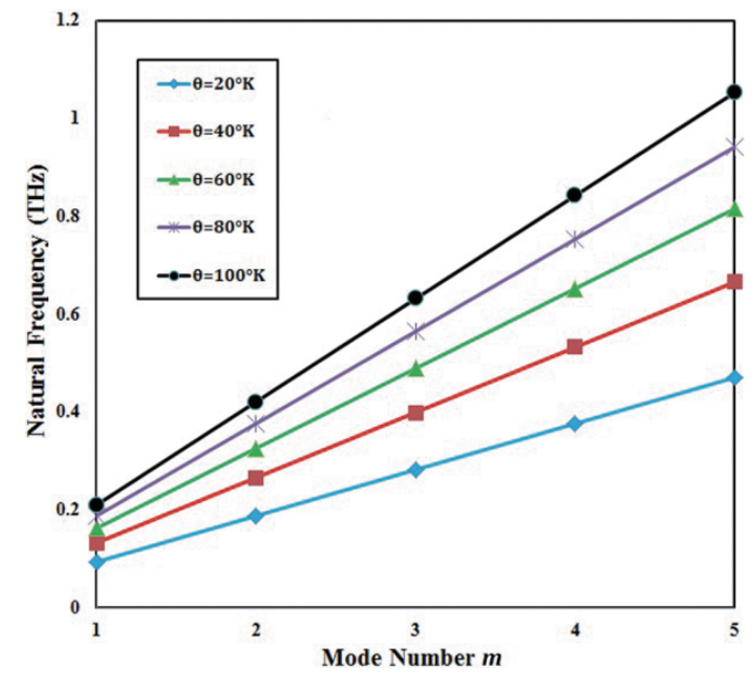

Fig. 10. Change of mode number $m$ on natural frequency for different temperature, $\left(\mu=0.5, L / d=5, K_{W}=K_{G}=0\right)$.

Case (3). $K_{G}=\mathbf{0}, \bar{N}=0$

In the absence of Pasternak foundation and thermal effect, we consider the effect of Winkler modulus in constitutive equation. The scale coefficients are taken as $e_{0} a \leqslant 2$ [35]. Figure 4 illustrates the effect of $K_{W}$ on natural frequency with different nonlocal parameter for different aspect ratio. It is clear that by increasing in nonlocal parameter $(\mu)$, the natural frequencies decrease but with increasing in aspect ratio $(L / d)$, the natural frequencies increase. In this case, by using Winkler foundation, the natural frequencies are slightly reduced with respect to case (2).

Figure 5 shows the effect of mode number $m$ on natural frequency in a fixed aspect ratio using Winkler modulus parameter. It is clear that by increasing in mode number, the natural frequencies decrease.

The effect of Winkler foundation $K_{W}$ on natural frequency with different aspect ratio is shown in Fig. 6. It is noted that natural frequency for short carbon nanotubes with higher Winkle modulus (e.g. $K_{W}=10^{5} \mathrm{~N} / \mathrm{m}^{2}$ ) are significantly affected by aspect ratio in comparison with lower Winkler modulus parameter (e.g. $K_{W}=10^{2} \mathrm{~N} / \mathrm{m}^{2}$ ). 


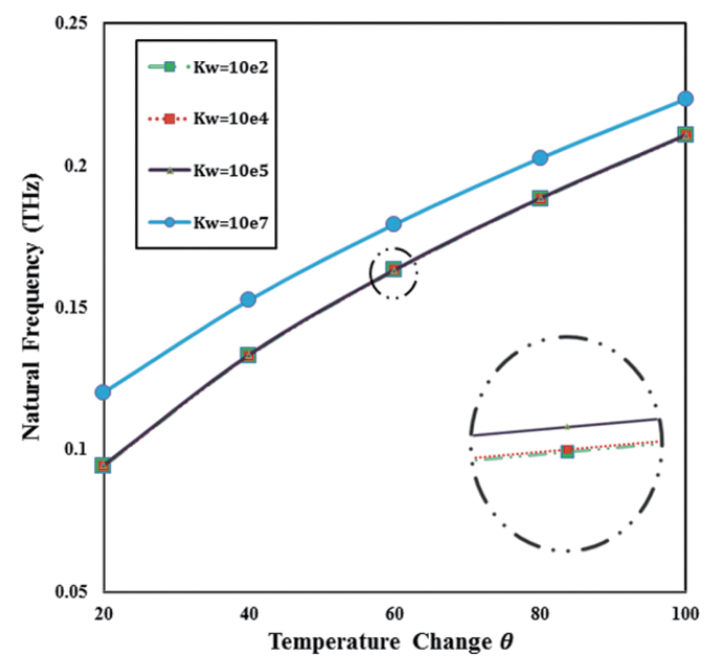

Fig. 11. Variation of natural frequency with temperature for different Winkler modulus $K_{W} \cdot\left(\mu=0.5, K_{G}=0, m=1\right)$.

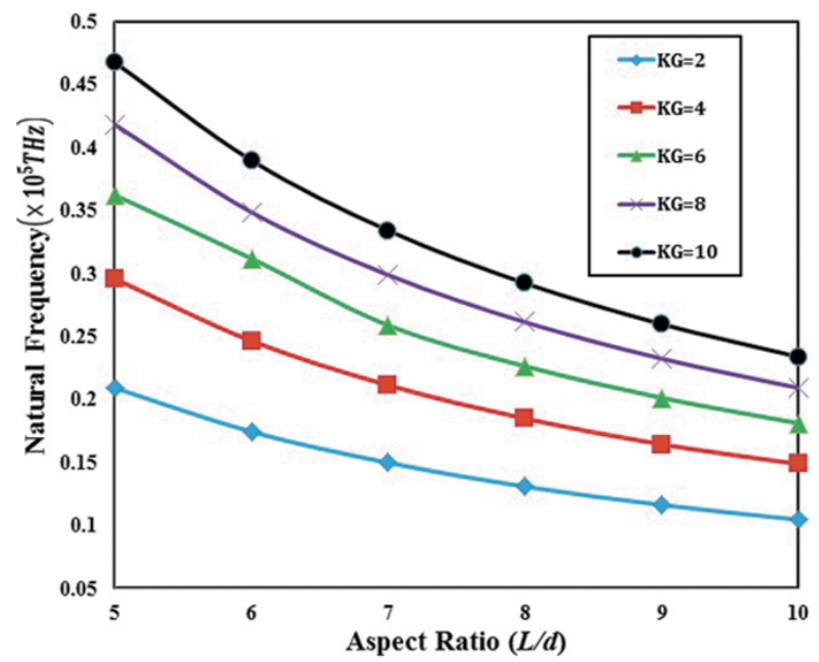

Fig. 12. Change of aspect ratio $L / d$ and effect of Pasternak foundation $K_{G}$ on frequency of a short-SWCNT, $\left(K_{W}=10^{2} \mathrm{~N} / \mathrm{m}^{2}, \theta=100^{\circ} \mathrm{K}\right.$, $m=1, \mu=0.5)$.

In general, natural frequencies increase due to increasing the Winkler modulus parameter. For $K_{W} \leqslant 10^{5} \mathrm{~N} / \mathrm{m}^{2}$, the plots are almost coincided on each other.

Case (4). $\bar{N}=0$

Effects of elastic foundation on the natural frequency of short single-walled carbon nanotubes with surrounding elastic medium modeled as Pasternak foundation are shown in Fig. 7. In this case, a value of $K_{W}=100$ is taken and the shear modulus parameter $K_{G}$ is varied from 0 to 10. These values are taken from Ref. [18] for the analysis of double-layered graphene sheets embedded in polymer matrix. It is assumed that the shearing layer stiffness of the foundation is one-tenth of the value of Winkler modulus [39]. Moreover, by increasing in aspect ratio, the natural frequencies increase significantly as compared with the case (3). As illustrated in Fig. 7, by increasing the shear modulus parameter $K_{G}$ in a constant aspect ratio, the natural frequencies of a short single-walled carbon nanotubes increase significantly and the frequencies are higher than the values in Fig. 4. The variation of natural frequencies with mode number for different shear modulus parameter is illustrated in Fig. 8. Here, nonlocal parameters and aspect ratio are assumed to be constant. Figure 8 depicts that with increasing in Pasternak constant, the natural frequencies increase.

Also, it is clear that the effect of mode number $m$ on natural frequencies is similar to shear modulus parameter. Therefore, it is noticeable that by increasing in shear modulus parameter $K_{G}$, the natural frequencies increase significantly.

Case (5)

This general cases is included all previous cases. In addition, the temperature effect has been considered here. It is reported that all the coefficients of thermal expansion for single walled carbon nanotube are negative at low temperature and are positive at high temperature [40]. Thus thermal expansion coefficient for short carbon nanotubes is taken as $+1.1 \times 10^{-6 \circ} \mathrm{K}$ [41]. In this case, the temperature changes are assumed to be uniform. Figures 9-12 are presented in order to review the effect of different parameter in frequency of a short-SWCNT. In Fig. 9, the effect of temperature change in natural frequency is considered. The Winkler and shear modulus parameter are equal to zero. It is clear that with increasing in temperature, the natural frequencies increase. In addition, the length-to-diameter parameter leads to decrease the natural frequencies.

The effect of mode number on natural frequency for different temperature change is shown in Fig. 10. Here, the nonlocal parameter and aspect ratio are constant. Therefore, with increasing in mode number $m$ and temperature change, the frequencies increase significantly. 
The simultaneously effects of Winkler modulus $K_{W}$ and temperature change on natural frequency are shown in Fig. 11. It is clear that by increasing in temperature, $\theta$, and Winkler modulus, $K_{W}$, the natural frequencies increase and for low Winkler modulus, the diagrams are almost coincided on each other and the frequencies response are identical. As compared with Fig. 9, using Winkler foundation leads to decrease natural frequencies. As mentioned before, the natural frequencies with higher Winkler modulus (e.g. $K_{W}=10^{7} \mathrm{~N} / \mathrm{m}^{2}$ ) are significantly affected by aspect ratio in comparison with lower Winkler modulus parameter (e.g. $K_{W}=10^{2} \mathrm{~N} / \mathrm{m}^{2}$ ).

Effects of aspect ratio along with Pasternak foundation and a fix nonlocal parameter are shown in Fig. 12. It is realized that with increasing in aspect ratio, natural frequencies decrease but in this case, with difference in temperature, natural frequency doesn't change significantly.

\section{Conclusions}

In this paper, the linear vibration characteristics of a short SWCNT embedded in Pasternak foundation in thermal environments were investigated. Analytical solution was used to solve the constitutive equations. The main results of this paper are obtained as follows:

An increase in nonlocal parameter leads to decrease the natural frequency. For a short SWCNT, with increasing the Winkler modulus in a constant aspect ratio, the natural frequencies increase. By comparing with the Winkler medium, for the Pasternak foundation, the frequency significantly increased. In addition, when a short SWCNT is subjected to various parameters simultaneously, the effect of Pasternak foundation on natural frequency is more significant. In this case, the effects of temperature change and Winkler foundation are negligible. For a short SWCNT, by increasing the temperature, in a constant nonlocal parameter, the natural frequencies increase. However, the frequency values are lower than the results obtained in case (2). Therefore, we realize that two-parameter elastic medium plays a very important role in frequencies of short SWCNT. Generally, it is concluded that the effect of Pasternak foundation on natural frequency is more significant as compared to the effects of thermal loading, Winkler modulus and nonlocal parameter and should be considered in vibration analysis of short carbon nanotubes.

\section{References}

[1] S. Iijima, Nature 354 (1991), 56-58.

[2] T.W. Ebbessen, Carbon Nanotubes Preparation and Properties, CRC Press, New York, 1997.

[3] E.V. Dirote, Trends in Nanotechnology Research, Nova Science Publishers, New York, 2004.

[4] S.C. Fang and W.J. Chang and Y.H. Wang, On the theory and design of acoustic resonators, Phys Letts A 371 (2007), 499-503.

[5] C.Y. Li and T.W. Chou, Single-walled carbon nanotubes as ultrahigh frequency nanomechanical resonators, Physical Review B 68 (2003), 073405.

[6] C.Y. Li and T.W. Chou, Mass detection using carbon nanotube-based nanomechanical resonators, Applied Physics Letters 84 (2004), 5246-5248.

[7] G. Yoon, C.Q. Ru and A. Mioduchowski, Vibration and instability of carbon nanotubes conveying fluid, Journal of Applied MechanicsTransactions of the ASME $\mathbf{6 5}$ (2005), 1326-1336.

[8] F. Scarpa and S. Adhikari, Uncertainty modeling of carbon nanotube terahertz oscillators, Journal of Non-Crystalline Solids 354 (2008), 4151-4156.

[9] W. Yang, X.L. Ma, H.T. Wang and W. Hong, The advancement of nanomechanics (continued), The Advancement of Mechanics 33 (2003), $175-185$.

[10] N. Pierard, A. Fonseca, Z. Konya, I. Willems, G. Van Tendeolo and J.B. Nagy, Production of short carbon nanotubes with open tips by ball milling, Chemical Physics Letters 335 (2001), 1-8.

[11] X. Xiao, J.N. Wang and F.S. Lian, Preparation an electrochemical performance of ultra-short carbon nanotubes, Journal of Power Sources 186 (2009), 194-200.

[12] M.J. Lopez, I. Cabria, N.J. March and J.A. Alonso, Structural and thermal stability of narrow and short carbon nanotubes and nanostrips, Carbon 43 (2005), 1371-1377.

[13] R.V. Seidel, A.P. Graham, J. Kretz, B. Rajasekharan, G.S. Duesberg, M. Liebau, E. Unger, F. Kreupl and W. Hoenlein, Sub-20 nm short channel carbon nanotube transistors, Nano Lett 5 (2005), 147-150.

[14] J. Yoon, C.Q. Ru and A. Mioduchowski, Vibration of an embedded multiwall carbon nanotube, Composites Science and Technology 63 (2003), 1533-1542.

[15] Y. Zhang, G. Liu and X. Han, Transverse vibration of double-walled carbon nanotubes under compressive axial load, Applied Physics Letter A 340 (2005), 258-266. 
[16] A.G. Arani, M.S. Zarei, M. Mohammadimehr, A. Arefmanesh and M.R. Mozdianfard, The thermal effect on buckling analysis of a DWCNT embedded on the Pasternak foundation, Physica E 43 (2011), 1642-1648.

[17] T. Murmu and S.C. Pradhan, Buckling analysis of single-walled carbon nanotube embedded in an elastic medium based on nonlocal elasticity and timoshenko beam theory and using DQM, Physica E 41 (2009), 1232-1239.

[18] K.M. Liew, X.Q. He and S. Kitipornchai, Predicting nanovibration of multi-layered graphene sheets embedded in an elastic matrix, Acta Materialia 54 (2006), 4229-4236.

[19] P. Soltani, P. Bahar and A. Farshidianfar, An efficient GDQ model for vibration analysis of a multiwall carbon nanotube on pasternak foundation with general boundary conditions, Journal of Mechanical Engineering Science 225 (2011), 1730-1741.

[20] P.L. Pasternak, Moscow, Gos Izd Lit Po Strait I Arkh (1954), $3^{\text {rd }}$. Edn, ed, (in Russian).

[21] S. Govindjee and J.L. Sackman, On the use of continuum mechanics to estimate the properties of nanotube, Solid State Communications 110 (1999), 227-230.

[22] G.F. Wang and X.Q. Feng, Timoshenko beam model for buckling and vibration of nanowires with surface effects, Journal of Physics D: Applied Physics 42 (2009), 1-5.

[23] A.C. Eringen, On differential equations of nonlocal elasticity and solutions of screw dislocation and surface waves, Applied Physics 4 (1983), 4703-4710.

[24] A.C. Eringen, Linear theory of nonlocal elasticity and dispersion of plane waves, International Journal of Engineering Science 10 (1972), 425-435.

[25] A.C. Eringen and D.G.B. Edelen, On nonlocal elasticity, International Journal of Engineering Science 10 (1972), $233-248$.

[26] H. Babaei and A.R. Shahidi, Small-scale effects on the buckling of quadrilateral nanoplates based on nonlocal elasticity theory using the Galerkin method, Arch Appl Mech 81 (2011), 1051-1062.

[27] X. Zhang, K. Jiao, P. Sharma and B.I. Yakobson, An atomistic and non-classical continuum field theoretic perspective of elastic interactions between defects (force dipoles) of various symmetries and application to graphene, J Mech Phys Solids 54 (2006), 2304-2329.

[28] W.H. Duan, C.M. Wang and Y.Y. Zhang, Calibration of nonlocal scaling effect parameter for free vibration of carbon nanotubes by molecular dynamics, J Appl Phys 101 (2007), 024305.

[29] Q. Wang, Wave propagation in carbon nanotubes via nonlocal continuum mechanics, J Appl Phys 98 (2005), 124301.

[30] H.S. Shen and C.L. Zhang, Torsional buckling and post buckling of double-walled carbon nanotubes by nonlocal shear deformable model, Compos Struct 92 (2010), 1073-1084.

[31] K.T. Chan and Y. Zhao, The dispersion characteristics of the waves propagating in a spinning single-walled carbon nanotube, Sci Chin Phys Mech Astron 54 (2011), 1854-1865.

[32] F. Khademolhosseini, P.A. Srikantha, N. Alireza and R. Nimal, Nonlocal continuum modeling and molecular dynamics simulation of torsional vibration of carbon nanotubes, IEEE Trans Nanotechnol 11 (2012), 00374.

[33] L. Wang and H.Y. Hu, Flexural wave propagation in single-walled carbon nanotube, Physical Review B 71 (2005), 195412.

[34] Y.Q. Zhang, G.R. Liu and X.Y. Xie, Free transverse vibrations of double-walled carbon nanotubes using a theory of nonlocal elasticity, Journal of Physical Review B 71 (2005), 195404.

[35] Q. Wang and C.M. Wang, The constitutive relation and small scale parameter of nonlocal continuum mechanics for modeling carbon nanotubes, Nanotechnology 18 (2007), 075702.

[36] J.N. Reddy and S.D. Pang, Nonlocal continuum theories of beams for the analysis of carbon nanotubes, Journal of Applied Physics $\mathbf{1 0 3}$ (2008), 1-16.

[37] J.Y. Hsieh, J.M. Lu, M.Y. Huang and C.C. Hwang, Theoretical variations in the Young's modulus of single-walled carbon nanotubes with tube radius and temperature: A molecular dynamics study, Nanotechnology 17 (2006), 3920-3924.

[38] Y.Q. Zhang, X. Liu and J.H. Zhao, Influence of temperature change on column buckling of multi-walled carbon nanotubes, Applied Physics Letters A 372 (2008), 1671-1681.

[39] H.S. Shen, J. Yang and S. Kitipornchai, Postbuckling of internal pressure loaded FGM cylindrical shells surrounded by an elastic medium, European Journal of Mechanics - A/Solids 29 (2010), 448-460.

[40] H. Jiang, R. Liu, Y. Huang and K.C. Hwang, Thermal expansion of single wall carbon nanotube, Journal of Engineering of Material and Technology 26 (2004), 265-270.

[41] X.H. Yao and Q. Han, Buckling analysis of multi-walled carbon nanotubes under torsional load coupling with temperature change, Journal of Engineering of Material and Technology 128 (2006), 419-428. 

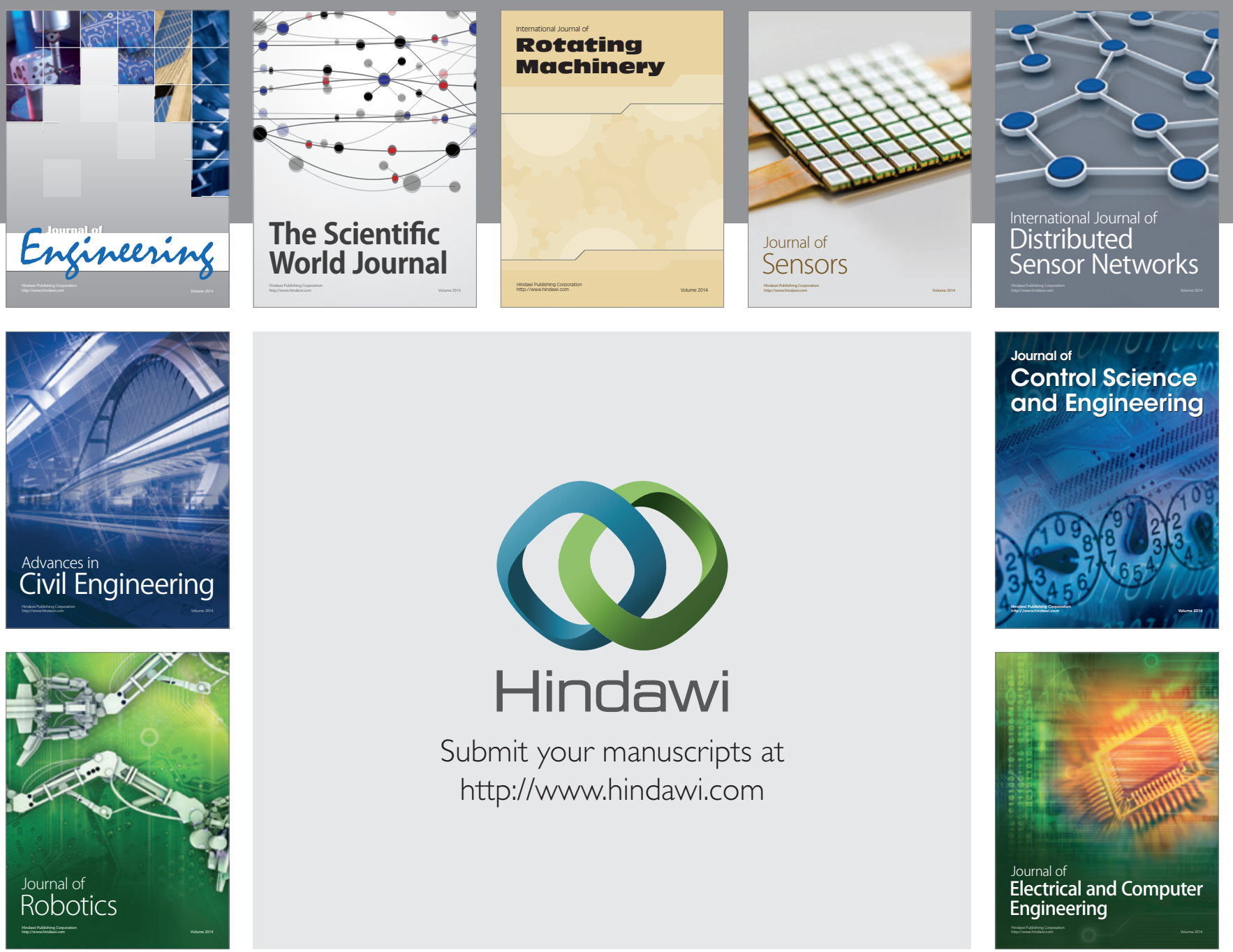

Submit your manuscripts at

http://www.hindawi.com
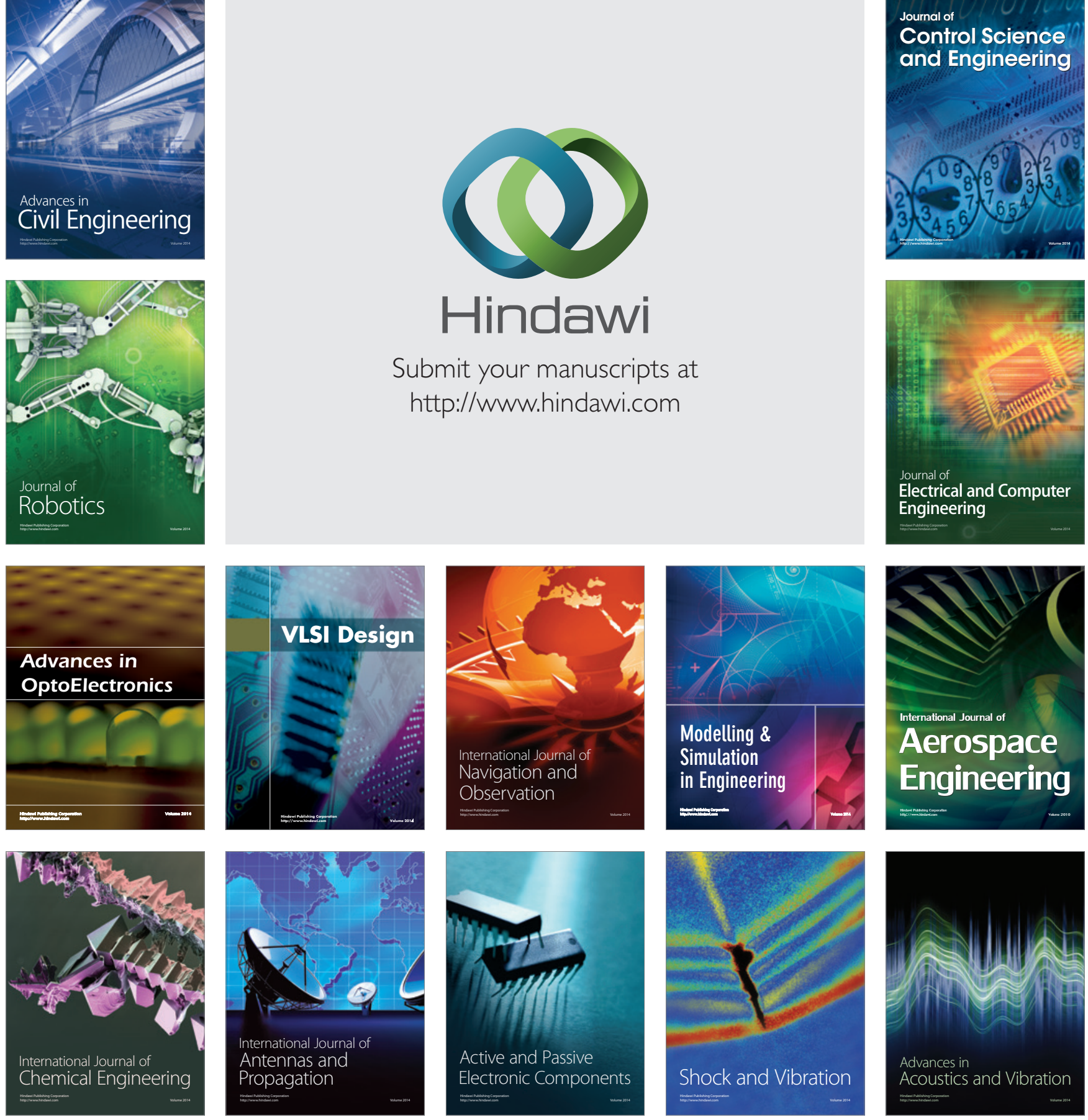